\title{
Dynamic Piston Function of the Mitral Annulus to Assess Early Left Ventricular Diastolic Filling: A Proof of Concept Study
}

\author{
Dagmar F. Hernandez-Suarez • Francisco López Menéndez • \\ Angel López-Candales
}

Received: December 27, 2018 / Published online: February 16, 2019

(C) The Author(s) 2019

\section{ABSTRACT}

Introduction: Early left ventricular (LV) filling has been described as an asymmetric toroidal vortex ring of blood entering the LV upon opening of the mitral valve. This phenomenon is in part responsible for cyclical changes in LV volumes during the cardiac cycle and also contributes to the apical and basal longitudinal displacements of the mitral annulus (MA). Although MA early diastolic ( $\left.e^{\prime}\right)$ velocities have been used to assess early LV filling characteristics, accurate distinction between normal aging and pathological diastolic dysfunction (DD) might be challenging at times.

Methods: In this pilot study, echocardiographic data from 60 consecutive patients were reviewed. The studied population was allocated into three groups based on the new American Society of Echocardiography guidelines for diastolic dysfunction classification. To better

Enhanced digital features To view enhanced digital features for this article go to https://doi.org/10.6084/ m9.figshare.7635920.

D. F. Hernandez-Suarez · A. López-Candales ( $\square)$ Cardiovascular Medicine Division, University of Puerto Rico School of Medicine, San Juan, PR, USA e-mail: angel.lopez17@upr.edu

F. López Menéndez

Division of Cardiovascular Health and Disease, University of Cincinnati College of Medicine,

Cincinnati, $\mathrm{OH}, \mathrm{USA}$ define LVDD, we based our interpretation of MA tissue Doppler imaging (TDI) signals on the well-described displacement pump mechanism of the MA plane functioning as a piston unit.

Results: Patients with normal diastolic function were younger ( $50 \pm 14$ years) than those with DD (group II: $69 \pm 8$ and group III: $63 \pm 17$ years $)(p<0.001)$ with a slight female predominance $(57 \%)$. As expected, volumetric variables as well as mitral inflow and MA TDI measures were significantly different among the three studied groups $(p<0.001)$ with the exception of the left atrial volume index. Interestingly, careful interrogation of the MA TDI signal revealed a distinctive appearance of a recoil signal right after the MA e' velocity only occurring in patients with normal LV diastolic function.

Conclusions: Identification of an early characteristic recoil signal occurring on the MA TDI right after the e' velocity seems to be useful in the characterization of LVDD. Additional prospective studies are now needed to validate its utility as an additional criterion to be used in LVDD.

Keywords: Diastolic function; Mitral annulus displacement; Recoil signal; Tissue Doppler imaging

\section{INTRODUCTION}

Assessment of left ventricular (LV) diastolic function has been traditionally based on 
concepts such as relaxation and stiffness, particularly when describing the mechanisms controlling early diastolic LV filling [1-4].

To simplify the complex nature of the mechanistic properties beyond the heart function as a pump, the interaction between LV volume and pressure changes has been approximated to the basal-to-apical longitudinal motion of the mitral annulus (MA). Being this last considered a reliable surrogate measurement for the assessment of both LV systolic and diastolic function $[5,6]$. Since this longitudinal motion of MA has been equated to that of a piston unit $[7,8]$, it is then obvious that LV systole and diastole are intrinsically related and mechanically coupled. In other words, the continuous longitudinal motion of the MA should be extremely valuable in the assessment of LV systole and diastole.

Despite the recent revision of LV diastolic function assessment guidelines [6], there are certain instances in which accurate interpretation of echo and Doppler data is somewhat insufficient when trying to categorize individuals with normal LV diastolic function from those with abnormal LV filling properties, particularly when not all required variables are in agreement.

Since our laboratory has previously characterized MA motion as it relates to LV diastole $[9,10]$, we have now focused our attention on studying early LV filling aside from the traditional measurement of e' velocity. Accordingly, we aimed to identify characteristic changes in the MA tissue Doppler imaging (TDI) recoil signal after the e' as a manifestation of the atrioventricular plane "piston pump" mechanism.

\section{METHODS}

For this proof of concept study, we analyzed echocardiographic data from 60 consecutive patients with a wide spectrum of medical and cardiac diseases referred for a complete echocardiographic. Patients were allocated into three groups based on the new American Society of Echocardiography (ASE) guidelines for diastolic dysfunction classification: [6, 11] normal LV diastolic function (group I) $(n=25)$, early relaxation abnormalities (group II) $(n=25)$, and pseudonormal pattern (group III) $(n=10)$. Aside for being a complete study including all echo Doppler variables, all patients had to be in normal sinus rhythm when data was acquired.

To accomplish the aim of this study, only TDI spectral signals from the lateral MA were used for analysis. This approach allowed us the interpretation of the most dynamic portion of the MA involved in longitudinal displacement.

Based on the previously described "piston pump" function of the MA, we coined the term, "recoil signal" as the spectral Doppler display seen above the baseline and occurring right after the MA TDI early e' velocity signal.

This article is based on previously conducted studies and does not involve any new studies of human or animal subjects performed by any of the authors. The University of Cincinnati, College of Medicine Institutional Review Board Committee approved data collection for this study (protocol number 12061302), and waived the need for consent. All procedures performed in studies involving human participants were in accordance with the 1964 Helsinki declaration and its later amendments or comparable ethical standards.

All patients were imaged with ultrasound systems equipped with multifrequency transducers and the study was performed using standard views with care taken to avoid foreshortening. From the apical four-chamber view, the lateral MA was interrogated. The resulting annular velocities by pulsed wave Doppler were recorded for 3-5 cardiac cycles at a sweep speed of $100 \mathrm{~mm} / \mathrm{s}$.

All echocardiographic and Doppler variables were acquired as recommended by current ASE guidelines $[6,11]$. Baseline characteristics were compared between groups using analysis of variance (ANOVA) for continuous variables, assuming equal variances and after testing for normality with Shapiro-Wilk test. A $p$ value $<0.05$ was considered statistically significant. All statistical analyses were performed using STATA version 14.2 (StataCorp, College Station, TX, USA).

\section{RESULTS}

The echocardiographic data of the study population is depicted in Table 1. Overall, patients 
Table 1 Echocardiographic data of the study population

\begin{tabular}{lcccr}
\hline Variables & $\begin{array}{l}\text { Group I } \\
\boldsymbol{n}=\mathbf{2 5}\end{array}$ & $\begin{array}{l}\text { Group II } \\
\boldsymbol{n}=\mathbf{2 5}\end{array}$ & $\begin{array}{l}\text { Group III } \\
\boldsymbol{n}=\mathbf{1 0}\end{array}$ & $\boldsymbol{p}$ value \\
\hline Age & $50 \pm 14$ & $69 \pm 8$ & $63 \pm 17$ & $<0.001$ \\
$\mathrm{BSA}$ & $2.0 \pm 0.3$ & $1.8 \pm 0.1$ & $1.9 \pm 0.3$ & 0.056 \\
LV mass index & $88 \pm 34$ & $94 \pm 42$ & $112 \pm 45$ & 0.295 \\
Left atrial volume index & $26 \pm 12$ & $31 \pm 12$ & $46 \pm 12$ & $<0.001$ \\
LV end diastolic volume & $106 \pm 41$ & $114 \pm 54$ & $195 \pm 72$ & $<0.001$ \\
LV end systolic volume & $37 \pm 26$ & $43 \pm 48$ & $143 \pm 65$ & $<0.001$ \\
LV ejection fraction & $68 \pm 14$ & $68 \pm 17$ & $27 \pm 14$ & $<0.001$ \\
MV E/A ratio & $1.4 \pm 0.5$ & $0.7 \pm 0.2$ & $1.4 \pm 0.3$ & $<0.001$ \\
MA TDI e' (lateral) & $12 \pm 3$ & $7 \pm 3$ & $4 \pm 2$ & $<0.001$ \\
MV E/MA TDI e' ratio & $8 \pm 2$ & $11 \pm 4$ & $27 \pm 10$ & $<0.001$ \\
PASP & $19 \pm 11$ & $20 \pm 9$ & $45 \pm 15$ & $<0.001$ \\
\hline
\end{tabular}

$B S A$ body surface area, $L V$ left ventricle, $M V$ mitral valve, $M A$ mitral annulus, $P A S P$ pulmonary artery systolic pressure

with normal diastolic function were younger (50 \pm 14 years) than those with diastolic dysfunction (group II: $69 \pm 8$ and group III: $63 \pm 17$ years) with a slight female predominance $(57 \%)$. There were no differences in terms of either body surface area (group I: $2.0 \pm 0.3$; group II: $1.8 \pm 0.1$; and group III: $1.9 \pm 0.3 \mathrm{~m}^{2}$ ) or LV mass index (group I: $88 \pm 34$; group II: $94 \pm 42$; and group III: $112 \pm 45 \mathrm{~g} / \mathrm{m}^{2}$ ).

Both group I and II had similar LV end systolic $(37 \pm 26$ versus $43 \pm 48 \mathrm{ml})$ and end diastolic $(106 \pm 41$ versus $114 \pm 54 \mathrm{ml})$ volumes but significantly smaller than group III $(143 \pm 65 \mathrm{ml}$ and $195 \pm 72 \mathrm{ml}$, respectively) patients. Consequently, group I and II had similar LV ejection fraction values $(68 \pm 14$ versus $68 \pm 17 \%)$, which were significantly higher than LV ejection values for group III $(27 \pm 14 \%)$ patients. Similarly, left atrial (LA) volume index values did not differ between group I and II $\left(26 \pm 12\right.$ and $31 \pm 12 \mathrm{ml} / \mathrm{m}^{2}$, respectively); however, LA volumes were significantly larger $\left(46 \pm 12 \mathrm{ml} / \mathrm{m}^{2}\right)$ in group III patients. Pulmonary artery systolic pressures were similar between group I and II $(19 \pm 11$ and $20 \pm 9 \mathrm{mmHg}$, respectively) while group III values were significantly higher $(45 \pm 15 \mathrm{mmHg})$.
As expected, not only the calculated mitral valve inflow E/A ratio was similar between group I and III $(1.4 \pm 0.5$ and $1.4 \pm 0.3$, respectively) while group II values were significantly lower $(0.7 \pm 0.2)$, but also the lateral MA TD $\mathrm{e}^{\prime}$ values were gradually smaller as the LV diastolic function deteriorated (group I: $12 \pm 3$; group II: $7 \pm 3$ and group III: $4 \pm 2 \mathrm{~cm} / \mathrm{s}$ ). Finally, the mitral valve inflow E velocity/MA TDI e' velocity ratio was higher with worse LV diastolic function (group I: $8 \pm 2$; group II: $11 \pm 4$ and group III: $27 \pm 10$ ).

Most importantly, appearance of a distinctive recoil signal occurring right after the MA e' velocity was only present in patients with normal LV diastolic function as seen in Fig. 1. None of the patients with either type I or II left ventricular diastolic dysfunction (LVDD) had this signal in the study population.

\section{DISCUSSION}

Having a basic understanding of the mechanisms that regulate cardiac dynamics have been a crucial quest to improve diagnosis and refine treatment alternatives that in turn result in better clinical outcomes. One of such pursuits 
Group I
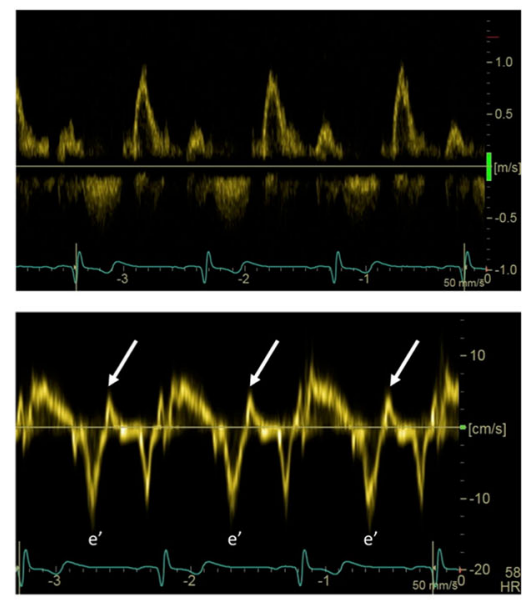

25 of 25 patients had this signal
Group II
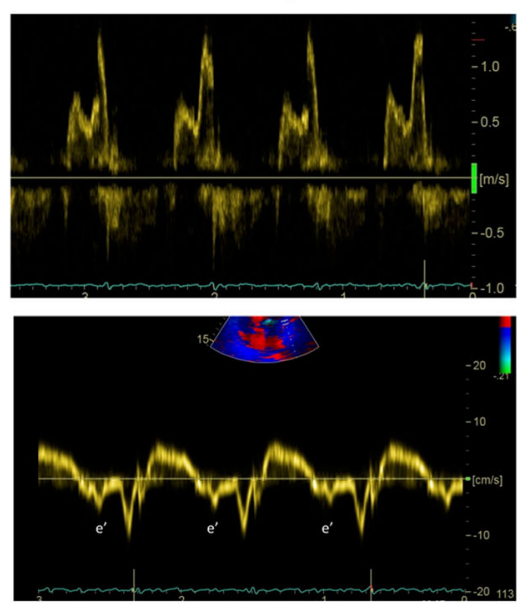

0 of 25 patients had the signal
Group III
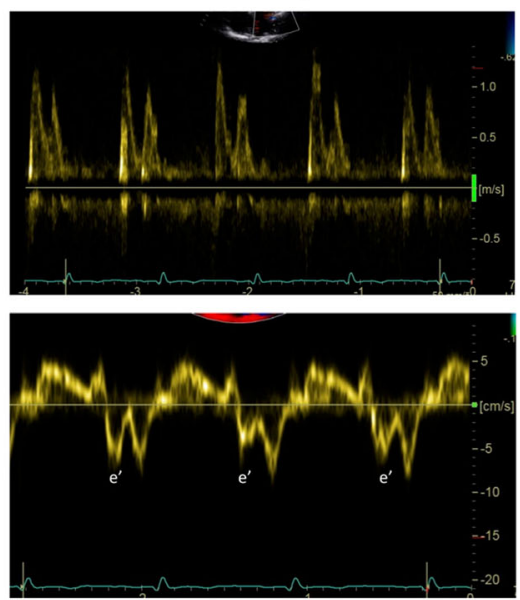

0 of 10 patients had the signal

Fig. 1 Recoil signal post MA TDI e' velocity (arrow)

has been the characterization of LV diastolic function. Even after the most recent publication of the revised LVDD guidelines [6], there are times where it remains difficult to characterize LVDD in some patients either since not all criteria are met or as result of technical issues when acquiring the echo Doppler data. Therefore, alternate approaches are still needed to improve LV diastolic function assessments.

Our results, although in a small sample of patients, reveal that careful interrogation of the lateral MA TDI spectral signal might be useful in this regard. This Doppler signal—already useful for measuring $\mathrm{e}^{\prime}$ velocities on the most dynamic portion of the MA-was also useful to study the proposed piston function of the MA. By using this portion of the MA, we have benefited from the uninterrupted view of the longitudinal motion of the annulus as seen from the fourchamber apical view. Through this approach, we were able to detect a recoil spectral signal right after the MA e' velocity in all patients that met the revised ASE guidelines for normal LV diastolic function and in none of the patients that were classified as either LVDD type I or type II using the same guidelines [6].

Gaining a better understanding of myocardial fiber's arrangement has certainly clarified systolic as well as diastolic LV function [12-17]. For example, LV twisting and untwisting have allowed a more comprehensive view of both systolic and early diastolic function, respectively. Work by Burn et al. [18] demonstrating reduced $L V$ untwisting was seen in patients with abnormal LV diastolic function, likely as a result of reduced suction gradients that are necessary to attain normal LV filling function.

From a mechanistic point of view, LV systole and diastole are not only driven by intracavitary pressure and flow differences, but also by longitudinal, radial and circumferential myocardial fibers contraction and relaxation. In this particular construct, basal-to-apical atrioventricular plane displacement occurs during systole contributing to both LV ejection and left atrial filling [19]. During ventricular diastole, as the atrioventricular plane returns to its initial position, the resultant hydraulic forces redistribute blood from the atria to the ventricle contributing to early LV filling. These longitudinal MA displacements have been approximated to the function of a piston [20]. This intricate coupling systolic and diastolic longitudinal displacement of the MA has been previously used to describe the association between LV systolic and diastolic dysfunction [21, 22].

The results of this pilot study using TDI to interrogate the lateral portion of the MA appear to be potentially helpful in clarifying LV diastolic filling properties. Specially, a characteristic recoil spectral signal right after the MA TDI e' 
velocity was only seen in patients classified as having normal LV diastolic function.

From 2009 to 2016, the ASE reduced the number of recommended criteria to assess LV diastolic function to only $4[6,23]$. Despite this, up to $15 \%$ of patients are deemed indeterminate as not all criteria fit. Therefore, this recoil spectral signal (easy to identify while assessing LV diastolic function through the MA TDI) might simply add confidence to the interpretation besides being a plausible mechanical explanation of the atrioventricular behavior during diastole.

However, the small sample size, absence of patients with arrhythmias, and retrospective nature of our study remain as potential limitations.

\section{CONCLUSIONS}

The findings of this pilot study intended to serve as a proof of concept of the potential piston function capability of the MA, aiding in the interpretation of the early LV filling properties of the LV. It seems that the presence of a characteristic recoil signal right after the occurrence of the MA e' velocity signal is indicative of normal diastolic function. We speculate that this signal simply indicates the presence of a very complaint LV. Based on these results, a larger validation prospective study is now necessary not only to determine whether this simple spectral signal could be useful in characterizing indeterminate patients based on current ASE LVDD assessment guidelines but also to discriminate between abnormal $\mathrm{LV}$ relaxation and aging.

\section{ACKNOWLEDGEMENTS}

We thank participants of the study.

Funding. This study and the article processing charges were funded by the National Institute of Health (NIH) Award Numbers U54MD007587, S21MD001830, R25MD007607, and TL1TR001434-3. Its contents are solely the responsibility of the authors and do not necessarily represent the official views of the National Institutes of Health.

Authorship. All named authors meet the International Committee of Medical Journal Editors (ICMJE) criteria for authorship for this manuscript, take responsibility for the integrity of the work as a whole, and have given final approval for the version to be published.

Disclosures. Dagmar F. Hernandez-Suarez, Francisco López Menéndez and Angel LópezCandales have nothing to disclose.

Compliance with Ethics Guidelines. This article is based on previously conducted studies and does not involve any new studies of human or animal subjects performed by any of the authors. The University of Cincinnati, College of Medicine Institutional Review Board Committee approved data collection for this study (protocol number 12061302), and waived the need for consent. All procedures performed in studies involving human participants were in accordance with the 1964 Helsinki declaration and its later amendments or comparable ethical standards.

Data Availability. The datasets analyzed during the current study are available from the corresponding author on reasonable request.

Open Access. This article is distributed under the terms of the Creative Commons Attribution-NonCommercial 4.0 International License (http://creativecommons.org/licenses/ by-nc/4.0/), which permits any noncommercial use, distribution, and reproduction in any medium, provided you give appropriate credit to the original author(s) and the source, provide a link to the Creative Commons license, and indicate if changes were made.

\section{REFERENCES}

1. Courtois M, Kovacs SJ Jr, Ludbrook PA. Transmitral pressure-flow velocity relation. Importance of 
regional pressure gradients in the left ventricle during diastole. Circulation. 1988;78:661-71.

2. Firstenberg MS, Smedira NG, Green-berg NL, et al. Relationship between early diastolic intraventricular pressure gradients, an index of elastic recoil, and improvements in systolic and diastolic function. Circulation. 2001;104:330-5.

3. Cheng C, Igarashi Y, Little W. Mechanism of augmented rate of left ventricular filling during exercise. Circ Res. 1992;70:9-19.

4. Rovner A, Greenberg NL, Thomas JD, Garcia MJ. Relationship of diastolic intraventricular pressure gradients and aerobic capacity in patients with diastolic heart failure. Am J Physiol Heart Circ Physiol. 2005;289:H2081-8.

5. Elnoamany MF, Abdelhameed AK. Mitral annular motion as a surrogate for left ventricular function: correlation with brain natriuretic peptide levels. Eur J Echocardiogr. 2006;7(3):187-98.

6. Nagueh SF, Smiseth OA, Appleton CP, et al. Recommendations for the evaluation of left ventricular diastolic function by echocardiography: an update from the American Society of Echocardiography and the European Association of Cardiovascular Imaging. J Am Soc Echocardiogr. 2016;29:277-314.

7. Ghosh E, Shmuylovich L, Kovacs SJ. Determination of early diastolic LV vortex formation time $\left(T^{*}\right)$ via the PDF formalism: a kinematic model of filling. Conf Proc IEEE Eng Med Biol Soc. 2009;2009:2883-6.

8. Maksuti E, Bjällmark A, Broomé M. Modelling the heart with the atrioventricular plane as a piston unit. Med Eng Phys. 2015;37:87-92.

9. Hernández Burgos PM, Lopez Menedez F, Candales MD, López-Candales A. Is mitral annular ascent useful in studying left ventricular function through left atrio-ventricular interactions? Indian Heart J. 2018;70(3):368-72.

10. Lopez-Candales A, Hernandez-Suarez DF, Lopez Menendez F. Mitral Annular dynamics and left ventricular diastole. Cardiol Res. 2017;8(5):228-31.

11. Lang RM, Badano LP, Mor-Avi V, et al. Recommendations for cardiac chamber quantification by echocardiography in adults: an update from the American Society of Echocardiography and the European Association of Cardiovascular Imaging. J Am Soc Echocardiogr. 2015;28(1):1-39.

12. Rademakers FE, Buchalter $\mathrm{MB}$, Rogers WJ, et al. Dissociation between left ventricular untwisting and filling. Accentuation by catecholamines. Circulation. 1992;85:1572-81.
13. Lester SJ, Tajik AJ, Nishimura RA, Oh JK, Khandheria BK, Seward JB. Unlocking the mysteries of diastolic function deciphering the Rosetta Stone 10 years later. J Am Coll Cardiol. 2008;51:679-89.

14. Sengupta PP, Tajik AJ, Chandrasekaran K, Khandheria B. Twist mechanics of the left ventricle: principles and application. J Am Coll Cardiol Imaging. 2008;1:366-76.

15. Notomi Y, Martin-Miklovic MG, Oryszak SJ, et al. Enhanced ventricular untwisting during exercise. A mechanistic manifestation of elastic recoil described by Doppler tissue imaging. Circulation. 2006;113:2524-33.

16. Dong SJ, Hees PS, Siu CO, Weiss JL, Shapiro EP. MRI assessment of $\mathrm{LV}$ relaxation by untwisting rate: a new isovolumic phase measure of tau. Am J Physiol Heart Circ Physiol. 2001;281:H2002-9.

17. Wang J, Khoury DS, Yue Y, Torre- Amione G, Nagueh SF. Left ventricular untwisting rate by speckle tracking echocardiography. Circulation. 2007;116:2580-6.

18. Burns AT, La Gerche A, Prior DL, MacIsaac AI. Left ventricular untwisting is an important determinant of early diastolic function. JACC Cardiovasc Imaging. 2009;2(6):709-16.

19. Steding-Ehrenborg K, Carlsson M, Stephensen S, Arheden H. Atrial aspiration from pulmonary and caval veins is caused by ventricular contraction and secures $70 \%$ of the total stroke volume independent of resting heart rate and heart size. Clin Physiol Funct Imaging. 2013;33:233-40.

20. Rushmer RF. Initial ventricular impulse. A potential key to cardiac evaluation. Circulation. 1964;29:268-83.

21. Willenheimer R, Israelsson B, Cline C, et al. Left atrioventricular plane displacement is related to both systolic and diastolic left ventricular performance in patients with chronic heart failure. Eur Heart J. 1999;20:612-8.

22. Vinereanu D, Nicolaides E, Tweddel AC, Fraser AG. Pure diastolic dysfunction is associated with longaxis systolic dysfunction. Implications for the diagnosis and classification of heart failure. Eur J Heart Fail. 2005;7:820-8.

23. Nagueh SF, Appleton CP, Gillebert TC, Marino PN, Oh JK, Smiseth OA, Waggoner AD, et al. Recommendations for the evaluation of left ventricular diastolic function by echocardiography. J Am Soc Echocardiogr. 2009;22(2):107-33. 